\title{
Calidad De Vida Y El Equilibrio Dinámico En El Adulto Mayor
}

\author{
Orlando David Mazón Moreno
}

Magíster en Cultura Física y Entrenamiento Deportivo

Docente de la Escuela Superior Politécnica de Chimborazo, Ecuador

Pablo Luis Lomas Badillo

Magíster en Cultura Física y Deportología

Docente de la Escuela Superior Politécnica de Chimborazo, Ecuador

\section{Danilo Ortiz Fernández.}

Magíster en Actividad Física

Docente de la Escuela Superior Politécnica de Chimborazo, Ecuador

\section{Santillan Obregon Rodrigo Roberto}

Magister en Entrenamiento Deportivo

Docente de la Escuela Superior Politécnica de Chimborazo, Ecuador

\section{Dennys Germán Palacios Valdiviezo}

Licenciado en Cultura Física y Entrenamiento Deportivo

Técnico Docente de la Escuela Superior Politécnica de Chimborazo, Ecuador

Doi: 10.19044/esj.2018.v14n14p110 URL:http://dx.doi.org/10.19044/esj.2018.v14n14p110

\begin{abstract}
The objective of the present investigation was to relate quality of life and dynamic balance in older adults. To this end, an intentional sample of older adults was taken from the gerontological centers of Chambo and Guano cantons, for which 35 people make up the control group (Chambo); while 71 people make up the intervention group (Guano). In order to reach the goal of the research, the quality of life was evaluated, through a pre-test called WHOQOL - BREF, followed by the Tinetti test that refers to the march balance, ending with the Timed get up and go test, which determines the mobility measures of people who can walk on their own. To the intervention group, an exercise program was applied to improve the dynamic balance that lasted ten weeks. Finally, by means of a post test, the entire sample was evaluated, which allowed us to analyze whether there was an incidence. In other words, a slight improvement in both dynamic balance and gait after ten weeks of intervention was evidenced.
\end{abstract}

Keywords: Quality of life, dynamic balance, older adults 


\section{Resumen}

El objetivo de la presente investigación, fue relacionar la calidad de vida y el equilibrio dinámico en el adulto mayor. Para ello, se tomó una muestra intencional de adultos mayores de los centros gerontológicos de los cantones de Chambo y Guano. De los cuales, 35 personas conforman el grupo de control (Chambo); mientras que 71 personas conforman el grupo de intervención (Guano). Para llegar a la meta, se evaluó la calidad de vida, a través de un pre test denominado WHOQOL - BREF, seguido del test Tinetti que hace referencia a la marcha - equilibrio, terminando con el test Timed get up and go, que determina las medidas de movilidad de las personas que pueden caminar por su propia cuenta. Al grupo de intervención, se aplicó un programa de ejercicios para mejorar el equilibrio dinámico que tuvo una duración de diez semanas. Finalmente, mediante un post test se evaluó toda la muestra que permitió analizar que si existió incidencia. Es decir se evidenció una mejora leve tanto en el equilibrio dinámico y la marcha después de las diez semanas de intervención.

Palabras-Claves: Calidad de vida, Equilibrio dinámico, adultos mayores

\section{Introducción:}

El argumento de la calidad de vida es extenso, pues en los últimos años se han tratado de realizar cambios significativos en la salud de la población, cuyo objetivo es mejorar necesariamente, la calidad de vida. De inicio es importante definir lo que es la calidad, lo que puede hacerse para los diferentes niveles. Calidad de vida: conjunto de condiciones que contribuyen a hacer agradable y valiosa la vida. (Peña, Terán, Moreno, \& Bazán, 2009, pág. 53)

El concepto de calidad de vida, ha comenzado a utilizarse cada vez más en el campo de las evaluaciones en salud, o como medida de bienestar. (Ursua \& Caqueo, 2012, p. 61)

Calidad de vida es un estado de satisfacción general, derivado de la realización de las potencialidades de la persona. Posee aspectos subjetivos y aspectos objetivos. Es una sensación subjetiva de bienestar físico, psicológico y social. Incluye como aspectos subjetivos la intimidad, la expresión emocional, la seguridad percibida, la productividad personal y la salud objetiva. Como aspectos objetivos el bienestar material, las relaciones armónicas con el ambiente físico y social y con la comunidad, y la salud objetivamente percibida. (Ardilla, 2009, p. 161)

La OMS (Organización Mundial de la Salud) indica que las caídas son la principal causa de morbimortalidad en el adulto mayor por falta del equilibrio dinámico, convirtiéndose en un grave problema de salud pública por las secuelas que estas producen. La prevalencia de caídas en el adulto mayor 
varía del $30 \%$ al $50 \%$ con una incidencia anual de 25 a 35\%. Del $10 \%$ al $25 \%$ de las caídas provocan fracturas en el adulto mayor por el poco equilibrio dinámico; el 5\% requiere hospitalización; mientras que las caídas son el 30\% de la causa de muerte en los mayores de 65 años. (Salud M. S., 2008)

En la encuesta "SABE Ecuador 2009 - 2010", se registra que de los adultos mayores de 65 a 74 años, el $38.7 \%$ presentaron caídas; de las cuales el $46.3 \%$ son mujeres, y un $29.8 \%$ hombres; mientras que en las personas mayores a 75 años, se registró el 40.6\%. (Freire, 2012)

A nivel mundial, las caídas son la segunda causa de muerte por lesiones accidentales o no intencionales. Se calcula que anualmente mueren en todo el mundo unas 424.000 personas debido a caídas y más del $80 \%$ de esas muertes se registran en países de bajos y medianos ingresos. Cada año se producen alrededor de 37.3 millones de caídas, cuya gravedad requiere atención médica. Los mayores de 65 años son quienes sufren más caídas mortales. (Cruz, González, López, Godoy, \& Pérez, 2014, p. 86)

Estudios internacionales, señalan que la tasa anual promedio de caídas en el grupo de 65 a 75 años de edad varía del $15 \%$ al $28 \%$ en personas sanas. Esta cifra se incrementa al $35 \%$ en mayores de 75 años de edad. (Calleja \& Lozano, 2010, p. 91)

Por consiguiente, las caídas y hasta los más ligeros resbalones representan un grave peligro de muerte, pérdida de autonomía y calidad de vida para los adultos mayores o de la tercera edad.

Las caídas constituyen uno de los síndromes geriátricos más importantes por su alta incidencia y por la elevada morbimortalidad. La Organización Mundial de la Salud (OMS) define a la caída como la consecuencia de cualquier acontecimiento que precipite una persona al suelo en contra de su voluntad. Las caídas ocurren a cualquier edad, siendo los niños y los adultos mayores los grupos con más incidencia. Sin embargo, las secuelas son muy distintas en ambos, siendo la mortalidad y la discapacidad altas en las personas mayores. Se calcula que un $7 \%$ de las visitas a emergencias que realizan los adultos mayores son debidas a una caída y de estas el 40\% terminan en una hospitalización. (OMS, 2011).

Es importante evitar los accidentes por caídas en los adultos mayores por su vulnerabilidad, por lo que se buscó la manera de combatir el problema mejorando el equilibrio dinámico, ya que gracias a él, el adulto mayor puede mantener su centro de gravedad y su sustentación evitando así caerse cuando se produce el movimiento.

El equilibrio es una habilidad imprescindible para la vida diaria, que requiere la compleja integración de información sensorial con respecto a la posición del cuerpo en relación con el entorno y la capacidad de generar respuestas motoras apropiadas para controlar el movimiento del cuerpo. 
Depende de las contribuciones de la visión, el sistema vestibular, la propiocepción y la fuerza muscular. (Melian, 2016, p. 47)

El ejercicio físico es un componente de estilo de vida saludable que en sus distintas facetas: gimnasia, deporte y la educación física constituyen actividades vitales para la salud, la recreación y el bienestar de la persona, la práctica de ejercicio físico, pueden hacer por la humanidad lo que no podrían alcanzar millones de médicos. (Soto, Cindy, 2014, p. 30)

Con ejercicios aeróbicos adecuados, aunque estos se empiezan a la edad de 60 años, se puede incrementar de uno a dos años la esperanza de vida, la independencia funcional, y ayudar a prevenir enfermedades. Se ha encontrado que las dos tercera parte de las personas con más de 60 años practican de manera irregular alguna actividad física o son totalmente sedentarios. Por causa de la inactividad, aparecen los riesgos de enfermedades crónicas como las cardiovasculares, la hipertensión, la diabetes, la osteoporosis y la depresión. Se puede mejorar significativamente la calidad de vida de una persona de la tercera edad dándole mayor flexibilidad, fuerza y volumen muscular, movilidad y mayor capacidad funcional aeróbica. (Barboza \& Alvarado, 2012, pág. 99)

La investigación solucionó el problema mediante un programa de intervención basado en ejercicios específicos para mejorar el equilibrio dinámico, que se llevó a cabo durante 10 semanas en el centro de atención integral del adulto mayor del cantón Guano y Centro gerontológico del cantón Chambo, con el objetivo de analizar y buscar una mejorar en la calidad de vida del adulto mayor.

\section{Metodología}

La investigación es de tipo Cuasi experimental, de corte transversal y de carácter cuali-cuantitativo, donde la muestra fueron los adultos mayores de los centros gerontológicos del cantón Chambo y cantón Guano con un total de 106 personas. Dividiendo para su estudio en dos grupos, 35 personas que es el grupo control perteneciente al centro gerontológico del Gobierno Autónomo Descentralizado GAD MUNICIPAL Chambo y el segundo grupo se forman con 71 personas de intervención pertenecientes al centro gerontológico Guano.

Tabla 1. Población y muestra grupo intervención y control

\begin{tabular}{|c|c|c|c|}
\hline \multicolumn{2}{|c|}{ Grupo } & \multirow{2}{*}{$\begin{array}{c}\text { Frecuencia } \\
26\end{array}$} & \multirow{2}{*}{$\begin{array}{c}\text { Porcentaje } \\
36,6\end{array}$} \\
\hline Intervención & Hombres & & \\
\hline \multirow{5}{*}{ Control } & Mujeres & 45 & 63,4 \\
\hline & Total & 71 & 100,0 \\
\hline & Hombres & 18 & 51,4 \\
\hline & Mujeres & 17 & 48,6 \\
\hline & Total & 35 & 100,0 \\
\hline
\end{tabular}

Fuente: Centros gerontológicos de los cantones Guano - Chambo

Elaboración: Dennys Palacios, 
Se utilizó la encuesta de calidad de vida WHOQOL- BREF que contiene un total de 26 preguntas, una pregunta para cada una de las 24 facetas contenidas en el WHOQOL-100 y dos preguntas globales: calidad de vida global y salud general. Cada ítem tiene 5 opciones de respuesta ordinales tipo Likert y todos ellos producen un perfil de cuatro áreas: salud física, psicológica, relaciones sociales y ambiente. El instrumento WHOQOL-BREF ofrece un perfil de calidad de vida, siendo cada dimensión o dominio puntuado de forma independiente. Cuanto mayor sea la puntuación en cada dominio, mejor es el perfil de calidad de vida de la persona evaluada. (OMS., 2004)

Se trabajó con el test "TINETTI", donde se utilizó la escala para medir la marcha y el equilibrio que determinó precozmente el riesgo de caída de un anciano durante el año siguiente a su aplicación. Generalmente se le pregunta al paciente si tiene miedo de caerse, ya que se ha comprobado que una respuesta afirmativa tiene un valor predictivo del $63 \%$ y del $87 \%$, si se trata de personas mayores con un grado apreciable de fragilidad. (Lázaro del Nogal, 2001)

La escala de Tinetti evaluó el equilibrio del paciente y se la realizó utilizando una silla sin brazos apoyada sobre la pared con la vigilia junto al paciente en posición de pie. Se evaluó la capacidad de equilibrio al sentarse en la silla, con el fin de determinar si lo hace con normalidad y sin necesidad de ayuda, o si se deja caer situándose fuera del centro de la silla; si es capaz de mantener la posición, si se mantiene erguido sin problemas o se separa del respaldo o se inclina levemente hacia un lado. (Lázaro del Nogal, 2001)

Se repitió el análisis, haciendo que el paciente se levante y comprobando que lo puede hacer sin ayuda; si necesita varios intentos antes de conseguirlo o si se balancea al realizar el esfuerzo. Así mismo, se evaluó el equilibrio inmediato al ponerse en pié; si se tambalea, mueve los pies, necesita apoyarse (bastón o andador) o se mantiene estable.

En la misma posición se realizó pruebas, como el sostener el equilibrio cambiando el peso de un pie a otro, o qué ocurre cuando el asistente tira ligeramente hacia atrás al paciente por la cintura (se cae o da algún paso hacia atrás). $\mathrm{O}$ si es capaz de mantener el equilibrio sobre un pie y durante cuánto tiempo lo hace. Se hace lo mismo en posición tándem y semitándem, al agacharse o al ponerse de puntillas o apoyar el peso sobre los talones. (Lázaro del Nogal, 2001)

La escala Tinetti evaluó la marcha del paciente, con el médico detrás del paciente y acompañándole en todo momento y asegurándose previamente que el suelo no tenga ningún tipo de irregularidad, teniendo en cuenta el material del suelo.

Se hizo caminar al paciente en trayectos de ida y vuelta, utilizando, si es el caso, el bastón de apoyo o el andador, con el fin de observar si hay algún tipo de vacilación al iniciar la marcha, si se desvía de la trayectoria rectilínea, 
si pierde el paso o el equilibrio, cómo da la vuelta para volver a realizar el mismo camino en sentido contrario o si es capaz de caminar normalmente a pesar de los obstáculos que se le pongan en el camino. (Lázaro del Nogal, 2001)

La puntuación máxima establecida para la prueba del equilibrio fue de 16 y para la marcha 12; sumando en total 28. Cuanto mayor es la puntuación final, mejor la funcionalidad del paciente y menor el riesgo de que pueda sufrir una caída, considerándose que por debajo de los 19 puntos hay un claro riesgo de caída que aumenta según desciende la puntuación. (Lázaro del Nogal, 2001)

Mediante el Test de movilidad "TIME GET UP AND GO", se evaluó el equilibrio funcional y se utilizó como indicador de riesgo de caídas. La evaluación fue ambulatoria debido al corto tiempo que requiere para ser aplicada. (Albala, 2010).

Para aplicar el test, se solicitó al paciente que se siente con su espalda apoyada en la silla y sus brazos descansando sobre los apoyabrazos de la silla. A continuación se le pidió que se ponga de pies y camine desde la silla a una distancia de 3 metros; luego gire y camine de vuelta a sentarse en la silla. (Albala, 2010)

El tiempo se determinó, desde que la persona comienza a levantarse de la silla hasta que retorna a la misma y se sienta. El paciente realizó una vez el test a forma de ensayo y luego 3 veces para ser evaluado, las mimas que fueron promediadas para obtener el resultado final. Los resultados son interpretados con los siguientes equivalentes de: 10 segundos es normal; de 11-20 segundos indica riesgo leve de caída y un puntaje $>20$ segundos indica alto riesgo de caída. (Albala, 2010)

Se aplicó el test WHOQOL - BREF en el escenario del pre en toda la población, tanto en el grupo de intervención del centro gerontológico (Guano), como en el grupo control del centro gerontológico (Chambo), procediendo a entregar las hojas a cada encuestado, de las cuales se obtuvo los diferentes resultados para luego aplicar el pre test de TINETTI, y por último se aplicó el pre test TIMED GET UP AND GO utilizando materiales como sillas, conos, etc.

Una vez obtenidos los resultados del pre test evaluados, se puso en marcha el programa de ejercicios con el fin de mejorar el equilibrio dinámico y la marcha; que se desarrollaron en diez semanas, los días lunes, viernes y sábados con 2 horas por día. En el que se realizaba el saludo, calentamiento, preparación de escenarios, etc. Se utilizó materiales como: pelotas, conos, tizas, cuerdas, costales, hula-hula, cronometro, silbato, contando con una excelente colaboración de parte de todo el personal.

Culminado el programa de ejercicios se procedió con la evaluación de los post test y encuestas en toda la población tanto en el grupo de intervención 
(Guano), como en el grupo control (Chambo) obteniendo resultados favorables y significativos.

Se plasmó un consentimiento informado que fue firmado por todos los participantes con las consideraciones éticas de HELSINKI 2000 en donde aceptaban formar parte de la investigación, así como también utilizar todos los datos que se recojan en la misma.

El análisis de datos se lo realizó a través de Microsoft Excel y el programa estadístico SPSS versión 22 IBM, USA.

\section{Resultados y discusión}

Tabla 1. Descriptiva centros gerontológicos Guano - Chambo

\begin{tabular}{|c|c|c|}
\hline \multicolumn{2}{|c|}{$\begin{array}{c}\text { WHOQOL - BREF Calidad de vida } \\
\text { Grupo Intervención (Guano) }\end{array}$} \\
\hline \multicolumn{2}{|c|}{} & $\begin{array}{c}\text { Cómo puntuaría su } \\
\text { calidad de vida }\end{array}$ \\
\hline \multirow{3}{*}{ Pre } & $\square$ & 2,65 \\
\cline { 2 - 3 } & $\sigma$ & 0,78 \\
\hline \multirow{3}{*}{ Post } & $\square$ & 2,68 \\
\cline { 2 - 3 } & $\sigma$ & 0,65 \\
\hline T Student & Sig & 0,53 \\
\hline
\end{tabular}

\begin{tabular}{|c|c|c|}
\hline \multicolumn{3}{|c|}{$\begin{array}{c}\text { WHOQOL - BREF Calidad de vida } \\
\text { Grupo Control (Chambo) }\end{array}$} \\
\hline \multicolumn{2}{|c|}{} & $\begin{array}{c}\text { Cómo puntuaría su } \\
\text { calidad de vida }\end{array}$ \\
\hline \multirow{3}{*}{ Pre } & $\square$ & 2,51 \\
\cline { 2 - 3 } Post & $\sigma$ & 0,82 \\
\cline { 2 - 3 } & $\square$ & 2,66 \\
\hline T Student & Sig & 0,87 \\
\hline
\end{tabular}

Fuente: Test WHOQOL - BREF Calidad de vida Grupo Intervención y Control

Elaborado por: Dennys Palacios

Después de haber aplicado la intervención a través de un programa de ejercicios en el centro gerontológico del cantón Guano-Chimborazo, en donde los encuestados seleccionaron en su mayoría la opción 2 que indica que la calidad de vida es Poco, $\square=2.68$, mientras que en el rango de calidad de vida del grupo control en el centro gerontológico del cantón Chambo-Chimborazo fue $\square=2.66$, mientras que en la investigación realizada por Sigüenza $M$, Sigüenza C, Sinche N., en la ciudad de Cuenca, donde se manifestaron en su mayoría por la opción 2 que indica que la calidad de vida es Poco, $\square=2.88$. Se pudo notar que la calidad de vida en sí de los dos cantones de la provincia de Chimborazo está en un rango Poco, con una mínima diferencia entre el grupo de control e intervención. 
Tabla 2. Descriptiva centros gerontológicos Guano - Chambo

\begin{tabular}{|c|c|c|c|c|c|}
\hline \multicolumn{3}{|c|}{$\begin{array}{l}\text { WHOQOL - BREF Calidad de vida } \\
\text { Grupo Intervención (Guano) }\end{array}$} & \multicolumn{3}{|c|}{$\begin{array}{l}\text { WHOQOL - BREF Calidad de vida } \\
\text { Grupo Control (Chambo) }\end{array}$} \\
\hline & & $\begin{array}{l}\text { Cuán satisfecho está } \\
\text { con su salud }\end{array}$ & & & $\begin{array}{l}\text { Cuán satisfecho está } \\
\text { con su salud }\end{array}$ \\
\hline \multirow[b]{2}{*}{ Pre } & $\square$ & 2,44 & \multirow[b]{2}{*}{ Pre } & $\square$ & 2,14 \\
\hline & $\sigma$ & 0,71 & & $\sigma$ & 0,94 \\
\hline \multirow[b]{2}{*}{ Post } & $\square$ & 2,54 & \multirow[b]{2}{*}{ Post } & $\square$ & 2,40 \\
\hline & $\sigma$ & 0,65 & & $\sigma$ & 0,77 \\
\hline T Student & Sig & 0,07 & T Student & Sig & 0,03 \\
\hline
\end{tabular}

Fuente: Test WHOQOL - BREF Calidad de vida Grupo Intervención y Control Elaborado por: Dennys Palacios

Posteriormente de haber aplicado la intervención en el centro gerontológico del cantón Guano-Chimborazo, en donde los participantes eligieron en su mayoría la opción 2 donde señala que la satisfacción de su salud es Poco, $\square=2.54$, mientras que en el rango de calidad de vida del grupo de control en el centro gerontológico del cantón Chambo-Chimborazo fue $\square=2.40$, mientras que en la investigación realizada por Sigüenza M, Sigüenza C, Sinche N., en la ciudad de Cuenca donde se manifestaron en su mayoría por la opción 2 que indica que la satisfacción de su salud es Poco, $\square=2.54$. Se pudo notar que la calidad de vida en sí de los dos cantones de la provincia de Chimborazo está en un rango Poco, con una mínima diferencia entre el grupo de control e intervención.

Tabla 3. Descriptiva centros gerontológicos Guano - Chambo

\begin{tabular}{|c|c|c|}
\hline \multicolumn{3}{|c|}{$\begin{array}{l}\text { TEST TINETTI MARCHA- } \\
\text { EQUILIBRIO GRUPO } \\
\text { INTERVENCIÓN (GUANO) }\end{array}$} \\
\hline & & Puntuación Total \\
\hline \multirow[b]{2}{*}{ Pre } & $\square$ & 17,69 \\
\hline & $\Sigma$ & 4,98 \\
\hline \multirow[b]{2}{*}{ Post } & $\square$ & 19,90 \\
\hline & $\Sigma$ & 4,78 \\
\hline T Student & Sig & $\mathbf{0 , 0 0}$ \\
\hline
\end{tabular}

\begin{tabular}{|c|c|c|}
\hline \multicolumn{3}{|c|}{ TEST TINETTI MARCHA- } \\
EQUILIBRIO \\
GRUPO CONTROL (CHAMBO) \\
\hline \multirow{3}{*}{ Pre } & & Puntuación Total \\
\cline { 2 - 3 } & $\sigma$ & 15,57 \\
\hline \multirow{3}{*}{ Post } & $\sigma$ & 4,55 \\
\cline { 2 - 3 } & $\sigma$ & 15,40 \\
\hline T Student & Sig & 4,60 \\
\hline
\end{tabular}

Fuente: Test TINETTI Marcha-Equilibrio grupo Intervención y Control

Elaborado por: Dennys Palacios

Luego de emplear la aplicación del programa de ejercicios en el centro gerontológico del cantón Guano-Chimborazo en donde los evaluados obtuvieron una puntuación global de $\square=19,90$ respecto al equilibrio y marcha, en cuanto al grupo control en el centro gerontológico del cantón ChamboChimborazo fue $\square=15,40$, mientras que en la investigación realizada por Ponce N., en la ciudad de Navarra-España, donde obtuvieron un promedio general de, $\square=15,22$. Se pudo observar que el equilibrio y marcha en los dos 
cantones de la provincia de Chimborazo existe una diferencia notable entre el grupo de control e intervención.

Tabla 4. Descriptiva centros gerontológicos Guano - Chambo

\begin{tabular}{|c|c|c|c|c|c|}
\hline \multicolumn{3}{|c|}{$\begin{array}{l}\text { TEST TIMED GET UP AND GO } \\
\text { GRUPO INTERVENCIÓN (GUANO) }\end{array}$} & \multicolumn{3}{|c|}{$\begin{array}{l}\text { TEST TIMED GET UP AND GO } \\
\text { GRUPO CONTROL (CHAMBO) }\end{array}$} \\
\hline & & Intentos Total & & & Intentos Total \\
\hline \multirow[b]{2}{*}{ Pre } & $\square$ & 31,15 & \multirow[b]{2}{*}{ Pre } & $\square$ & 42,43 \\
\hline & $\sigma$ & 21,69 & & $\sigma$ & 13,56 \\
\hline \multirow[b]{2}{*}{ Post } & $\square$ & 30,03 & \multirow[b]{2}{*}{ Post } & $\square$ & 42,76 \\
\hline & $\sigma$ & 20,09 & & $\sigma$ & 13,76 \\
\hline T Student & Sig & $\mathbf{0 , 0 0}$ & T Student & Sig & 0,12 \\
\hline
\end{tabular}

Fuente: Test TIMED GET UP AND GO grupo intervención y Control

Elaborado por: Dennys Palacios

Una vez empleado el programa de ejercicios en el centro gerontológico del cantón Guano-Chimborazo, en donde los participantes obtuvieron una puntuación global de $\square=30.03$ con respecto a las medidas de movilidad, en cuanto al grupo control en el centro gerontológico del cantón ChamboChimborazo fue $\square=42.76$, mientras que en la investigación realizada por Manzillas E, Valenzuela J, Escobar M, en Chile donde obtuvieron un promedio general de, $\square=11,03$. Se pudo observar que las medidas de movilidad en los dos cantones de la provincia de Chimborazo existe una diferencia sustancial entre el grupo de control e intervención.

\section{Conclusion}

- A través de la aplicación del TEST WHOQOL - BREF se logró obtener datos referentes a la calidad de vida en los grupos de intervención y control que representó el escenario del pre o el escenario donde aún no se aplicó el programa de ejercicios para mejorar la calidad de vida de los adultos mayores.

- $\quad$ Con la aplicación del test TINETTI marcha-equilibrio en el grupo de intervención y control se logró determinar la movilidad y marcha en los adultos mayores antes de aplicar el programa de ejercicios.

- $\quad$ Con el diseño y aplicación el programa de ejercicios para adultos mayores enfocados en la calidad de vida y la marcha equilibrio. Se logró preparar el escenario para que la muestra sea sometida nuevamente a los test de WHOQOL - BREF y TINETTI con el fin de comparar los resultados con los resultados del pre test.

- $\quad$ El nivel de calidad de vida de los adultos mayores del centro gerontológico de Guano - Ecuador se encuentra en un rango Poco, con $\square=$ 2,68; mientras que el nivel de equilibrio dinámico de los adultos mayores del 
centro gerontológico de Guano - Ecuador arrojó una $\square=19,90$ post intervención con un $\mathrm{p}=0,00$.

- $\quad$ Los niveles de calidad de vida se mejoraron $(\mathrm{p}=0,53)$, y en el equilibrio dinámico se evidenció una mejora significativa $(\mathrm{p}=0,00)$

\section{Recomendaciones}

- Se recomienda, realizar un trabajo integral en los adultos mayores, incorporando actividades acorde a su edad evolutiva

- Se recomienda, continuar con actividades lúdicas enfocados a la mejora del equilibrio dinámico

- Se recomienda, seguir mejorando el equilibrio dinámico con el objetivo de evitar caídas en los adultos mayores, y no afectar su calidad de vida.

\section{References:}

1. Albala, C. (2010). Universidad de Chile.

2. Ardilla, Ruben. (2009). CALIDAD DE VIDA: UNA DEFINICIÓN INTEGRADORA. Revista Latinoamericana de Psicología, 161.

3. Barboza, R., \& Alvarado, D. (2012). Beneficios del Ejercicio y la Actividad Física en la Tercera Edad. Revsta educación, 99-103.

4. Calleja, O., \& Lozano, D. (2010). Prevención y Atención de las Caídas en la Persona Adulta Mayor. Guía de Consulta para el Médico de Primer Nivel de Atención, 91-110.

5. Cruz, E., González, M., López, M., Godoy, I., \& Pérez, M. (2014). Caídas: revision de nuevos conceptos. Hospital Universitario Pedro Ernesto, 86-95.

6. Freire, W. (03 de Agosto de 2012). SABE. Obtenido de SABE: http://www.gerontologia.org/portal/archivosUpload/EcuadorEncuesta-SABE-presentacion-resultados.pdf

7. Lázaro del Nogal M. . (2001). Lázaro del Nogal M. Evaluación del anciano que sufre caídas de repetición. . Madrid; Fundación Mapfre Medicina.

8. Melian, C. (2016). Trastornos del equilibrio en el adulto mayor. REVISTA FASO AÑO 23 - Suplemento vestibular $2^{\circ}$ Parte - , 47.

9. OMS. (2011). La actividad fisica en adultos mayores. Revista OMS, 25-48.

10. OMS. (2004). Calidad de vida de adultos mayores pobres de viviendas básicas:Estudio comparativo mediante uso de WHOQOL-BREF. The World Health Organization Quality of Life (WHOQOL)-BREF World Health Organization, 325-333.

11. Peña, B., Terán, M., Moreno, F., \& Bazán, M. (2009). Autopercepción de la calidad de vida del adulto mayor en la Clínica de Medicina 
Familiar Oriente del ISSSTE. Revista de Especialidades MédicoQuirúrgica, 53-61.

12. Salud, O. M. (18 de Marzo de 2017). El telegrafo. Obtenido de El telegrafo: http://www.eltelegrafo.com.ec/noticias/palabramayor/17/caidas-restan-calidad-de-vida-al-aduto-mayor

13. Soto, Cindy. (2014). Valoración del Equilibrio y Marcha en Adultos Mayores que participan y no, en un Programa de Ejercicio Físico, en el Hospital San Juan De Lurigancho-Enero 2014. E.A.P. DE TECNOLOGÍA MEDICA, 30.

14. Ursua, A., \& Caqueo, A. (2012). Calidad de vida: Una revisión teórica del concepto. Scielo, 61. 